\title{
One versus two years of elastic compression stockings for prevention of post-thrombotic syndrome (OCTAVIA study): randomised controlled trial
}

\author{
G C Mol,1,2,3 M A van de Ree, ${ }^{1}$ F A Klok,, ${ }^{4}$ M J A M Tegelberg, ${ }^{5}$ F B M Sanders, ${ }^{6}$ S Koppen, ${ }^{7}$ \\ O de Weerdt, ${ }^{8}$ T Koster, ${ }^{9}$ M M C Hovens, ${ }^{10}$ H A H Kaasjager, ${ }^{2}$ R E Brouwer, ${ }^{11}$ E Kragten, ${ }^{11}$ \\ C G Schaar, ${ }^{12}$ W Spiering, ${ }^{3}$ W P Arnold, ${ }^{13}$ D H Biesma, 8 M V Huisman ${ }^{4}$
}

For numbered affiliations see end of article.

Correspondence to: M V Huisman m.v.huisman@lumc.nl

Cite this as: BMJ 2016;353:i2691 http://dx.doi.org/10.1136/bmj.i2691

Accepted: 26 April 2016

\section{ABSTRACT}

OBJECTIVE

To study whether stopping elastic compression stockings (ECS) after 12 months is non-inferior to continuing them for 24 months after proximal deep venous thrombosis.

DESIGN

Multicentre single blind non-inferiority randomised controlled trial.

SETTING

Outpatient clinics in eight teaching hospitals in the Netherlands, including one university medical centre.

\section{PARTICIPANTS}

Patients compliant with compression therapy for 12 months after symptomatic, ultrasound proven proximal deep venous thrombosis of the leg.

INTERVENTIONS

Continuation or cessation of ECS 12 months after deep venous thrombosis.

\section{MAIN OUTCOME MEASURES}

The primary outcome was the incidence of postthrombotic syndrome 24 months after diagnosis of deep venous thrombosis, as assessed by the standardised Villalta scale in an intention to treat analysis. The predefined non-inferiority margin was $10 \%$. The main secondary outcome was quality of life (VEINES-QOL/Sym).

\section{RESULTS}

518 patients compliant with ECS and free of postthrombotic syndrome were randomised one year after diagnosis of deep venous thrombosis to stop or continue ECS therapy for another year. In the stop-ECS group, 51 of 256 patients developed post-thrombotic syndrome, with an incidence of $19.9 \%$ (95\% confidence interval $16 \%$ to $24 \%$ ). In the continue-ECS

\section{WHAT IS ALREADY KNOWN ON THIS TOPIC}

Elastic compression stockings (ECS) are used for the prevention of post-thrombotic syndrome after deep venous thrombosis, but the optimal duration of compression therapy is unknown

Current guidelines suggest 24 months of ECS use, but recent studies have questioned this strategy with suggestions of shorter duration of ECS therapy

\section{WHAT THIS STUDY ADDS}

In patients with excellent adherence to ECS use, stopping ECS one year after deep venous thrombosis was not non-inferior to continuing ECS in preventing postthrombotic syndrome

ECS therapy should ideally be continued for 24 months after deep vein thrombosis group, 34 of 262 patients developed post-thrombotic syndrome (incidence $13.0 \%, 9.9 \%$ to $17 \%$ ), of whom $85 \%$ used ECS six or seven days a week during the study period, for an absolute difference of $6.9 \%$ (95\% confidence interval upper limit 12.3\%). Because the upper limit of the $95 \%$ confidence interval exceeds the predefined margin of $10 \%$, non-inferiority was not reached. The number needed to treat to prevent one case of post-thrombotic syndrome by continuing ECS was 14 (95\% confidence interval lower limit 8). Quality of life did not differ between the two groups.

\section{CONCLUSION}

Stopping ECS after one year in compliant patients with proximal deep venous thrombosis seemed not to be non-inferior to continuing ECS therapy for two years in this non-inferiority trial.

\section{TRIAL REGISTRATION}

Netherlands Trial Register NTR1442.

\section{Introduction}

Post-thrombotic syndrome is a common and chronic complication of deep venous thrombosis of the leg that may develop in one of every two to three patients despite optimal anticoagulant treatment. ${ }^{12}$ Most patients with post-thrombotic syndrome develop the first symptoms within two years after diagnosis of deep venous thrombosis, the majority within the first year. ${ }^{3-6}$ Symptoms of post-thrombotic syndrome may range from skin changes through pain or mild swelling to badly controlled oedema and chronic leg ulcers, often needing high intensity medical treatment. Patients with post-thrombotic syndrome also report significant disability and impaired quality of life, which imposes a considerable economic burden on patients and healthcare systems. ${ }^{27-9}$

Strategies for prevention of post-thrombotic syndrome involve use of thromboprophylaxis in patients at significant risk for deep venous thrombosis and providing anticoagulation of appropriate intensity and duration once it is established. ${ }^{2}$ In addition, the results of two relatively small randomised controlled trials have suggested that continuous use of graded elastic compression stockings (ECS) for at least two years provides a $50 \%$ reduction in the incidence of post-thrombotic syndrome compared with no stockings, with a number needed to treat of four patients. ${ }^{46}$ This strategy has been implemented in international guidelines and daily clinical practice. The effectiveness of ECS in preventing post-thrombotic syndrome is debated, however, as a placebo effect in the trials cannot be excluded. 
The positive effect of ECS was recently challenged by the SOX trial, which could not show any benefit of ECS over placebo stockings in the prevention of post-thrombotic syndrome. ${ }^{10}$ Notably, this trial had very poor adherence; only $56 \%$ of patients were using the study stockings for three days a week or more at the end of the study, and a clear trend to less frequent use of the stocking was apparent over the two year follow-up period. The SOX trial strongly underlined the major problem of non-adherence, probably due to local irritation and cosmetic reasons. ${ }^{11}$

Pragmatic considerations have led to the suggestion to discontinue compression therapy earlier, although evidence supporting this practice is lacking. Both incidence of post-thrombotic syndrome and adherence to ECS decrease over time. ${ }^{4610}$ A prospective management cohort study with 125 participants in Maastricht, the Netherlands, suggested that individually tailored duration of ECS based on Villalta scores and ultrasound findings can be effective and shorten duration of ECS use. ${ }^{12}$ This strategy is being studied in a randomised clinical trial. ${ }^{13}$ We hypothesised that ECS therapy for 12 months would be non-inferior to prevention of post-thrombotic syndrome with standard care-that is, ECS therapy for 24 months. Accordingly, the aim of our study was to evaluate the effect of ECS therapy for a period of 12 months versus 24 months after a diagnosis of proximal deep venous thrombosis on the occurrence of post-thrombotic syndrome and disease specific quality of life.

\section{Methods}

\section{Study patients}

The study was conducted in eight teaching hospitals in the Netherlands, including one university medica centre. Between February 2009 and September 2013, consecutive patients in the outpatient clinic with a recent diagnosis of deep venous thrombosis were invited to participate in this trial. Patients were eligible for inclusion if they had been diagnosed as having a symptomatic, compression ultrasound proven, proximal deep venous thrombosis of the leg (popliteal or more proximal deep leg veins) less than one year previously; had been treated with anticoagulants in accordance with applicable guidelines; had received graduated ECS class III (34-46 mm Hg); and reported strict compliance, defined as using the stockings at

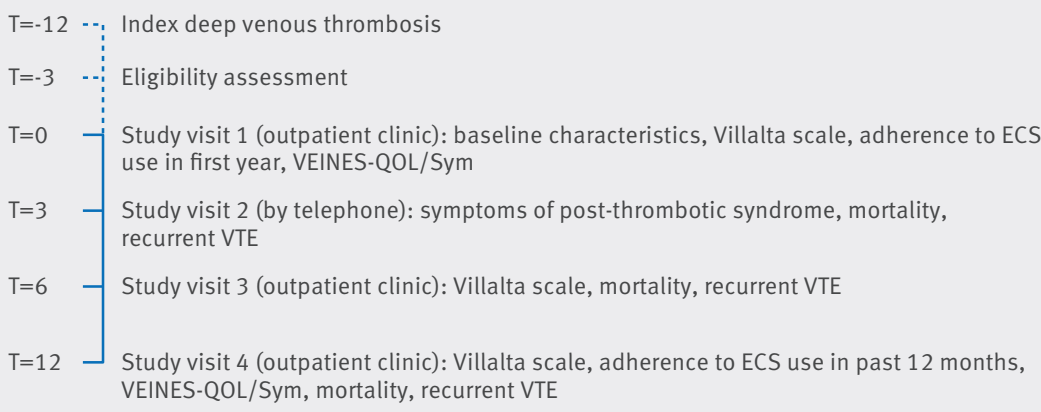

Fig 1 Study design (time in months). ECS=elastic compression stockings; VTE=venous thromboembolism least six days a week. Exclusion criteria were recurrent ipsilateral deep venous thrombosis; calf vein thrombosis (defined as thrombosis only below the level of the popliteal vein); post-thrombotic syndrome diagnosed in the first year after deep venous thrombosis before study inclusion, defined as a Villalta score of 5 points or more; ${ }^{14-16}$ use of ECS before the index deep venous thrombosis diagnosis for other reasons; a contraindication to wearing compression stockings, such as arterial insufficiency or allergy; life expectancy less than six months; comorbid conditions or geographical inaccessibility precluding return for follow-up visits; and no informed consent.

\section{Randomisation and blinding}

One year after diagnosis of the index deep venous thrombosis, all eligible patients visited the outpatient clinic for definite screening of inclusion and exclusion criteria, baseline visit, and randomisation. Randomisation used an online randomisation module provided by the Dutch Julius Centre for Health Sciences and Primary Care in Utrecht, the Netherlands, which ensured concealed allocation. We used stratification for sex, study centre, and timing of randomisation per season of the year (to correct for possible seasonal differences in use of ECS). After randomisation, the study participants received a written alert with their allocation. Treatment allocation was masked from study personnel during follow-up. Patients who were allocated to standard care were asked not to wear the stocking on the day of follow-up, and all study participants were instructed not to reveal their allocation at study visits.

\section{Procedures and outcome assessment}

Patients were randomly assigned to either continue or stop wearing active graduated ECS one year after the index deep venous thrombosis. Patients allocated to the stop-ECS group stopped using the ECS on the day they received the written alert of randomisation. ECS were used only during daytime hours, and no other specific form of calf muscle exercise (or instructions to do so) was performed. Follow-up visits were scheduled at three, six, and 12 months from the baseline visit (fig 1). At baseline, a clinical history was taken detailing the presence of risk factors for venous thrombosis, the applied anticoagulation treatment, the first time of ECS use, adherence to ECS in the first year after diagnosis of deep venous thrombosis, medical history, and current use of (anticoagulant) drugs. The extent and location of the deep venous thrombosis were verified with the original compression ultrasound reports.

The primary endpoint was the incidence of post-thrombotic syndrome at the end of the follow-up period-that is, post-thrombotic syndrome occurring in the second year after a diagnosis of deep venous thrombosis. Post-thrombotic syndrome was scored using the Villalta scale assessed with the aid of a full colour visual guide, ${ }^{1015}$ with post-thrombotic syndrome defined as a score of 5 points or more or the presence of a venous ulcer. ${ }^{1416}$ The Villalta scale was preferably evaluated in the afternoon by a maximum of two 
experienced outcome assessors for each participating centre. The presence of post-thrombotic syndrome was evaluated at the outpatient clinic during the baseline visit and after six and 12 months. At the three month visit, patients were contacted by telephone to evaluate them (using a checklist) for new signs of post-thrombotic syndrome. If any signs were present, the phone call was followed by clinical evaluation.

Secondary endpoints included adherence to ECS use during study follow-up as reported by the patients at the 12 month visit, recurrent deep venous thrombosis in the same leg as the index event, death, and within patient changes in quality of life over the study period as assessed by the VEINES-QOL/Sym questionnaire that was recorded at baseline and at the 12 month visit. ${ }^{18} 18$ The outcome of the VEINES-QOL/Sym questionnaire is reported in two dimensions-overall quality of life (VEINES-QOL; 25 items) and symptom severity (VEINES-Sym; 10 items). Both scales theoretically lie between 0 and 100 (visual analogue scale) with a higher score indicating a higher quality of life. ${ }^{18-20}$ We defined dedicated ECS use as use of the ECS for at least six days a week, moderate use as four to five days a week, and poor use less than four days a week. We defined recurrent ipsilateral deep venous thrombosis as a new non-compressible venous segment compared with the original compression ultrasound images of the index deep venous thrombosis or a pronounced increase in vein diameter on compression..$^{21-23}$

\section{Statistical analysis}

The trial was designed to determine whether 12 months of ECS use was non-inferior to 24 months, with a predefined non-inferiority margin of $10 \%$, meaning that the upper boundary of the $95 \%$ confidence interval of the absolute difference between the primary endpoint in

Patients assessed for eligibility $(n=3603)$

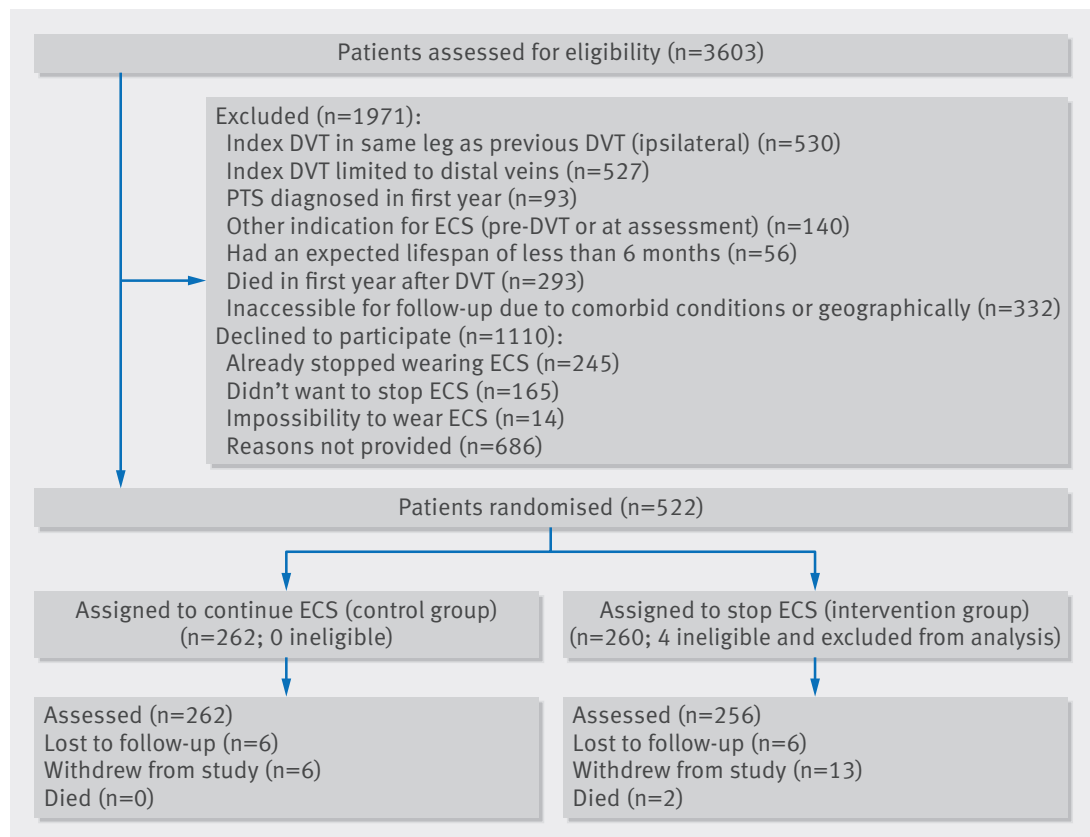

Fig 2 | Flow chart of study. DVT=deep venous thrombosis; ECS=elastic compression stockings; PTS=post-thrombotic syndrome the two study groups would be lower than $10 \%$. We agreed on this margin as a clinically relevant maximum increase in diagnoses of post-thrombotic syndrome that would be acceptable to change the current recommendation of ECS duration from 24 to 12 months. We analysed efficacy according to an intention to treat principle to assess the incidence of post-thrombotic syndrome. We determined the sample size on the basis of an expected rate of the primary efficacy outcome in the reference group (continue-ECS group) of 2-3\%, derived from cohorts in previous studies, in which the incidence of post-thrombotic syndrome in the second year after deep venous thrombosis was about that percentage, ${ }^{46}$ and the requirement to achieve $80 \%$ power to detect the predefined non-inferiority margin at a one sided $\alpha$ level of 0.05 . Assuming a loss to follow-up of $10 \%$, including deaths and recurrent ipsilateral deep venous thrombosis events, the total study population was set at 516 people.

We additionally did a per protocol analysis of the primary endpoint. We then did extended Cox regression analyses on the primary outcome with adjustment for adherence and baseline covariates-that is, age, sex, body mass index, unprovoked or secondary deep venous thrombosis, extent of the index deep venous thrombosis, baseline Villalta score, study site, and the use of oral anticoagulants during the study period.

For secondary endpoints, we compared the occurrence of recurrent ipsilateral deep venous thrombosis and overall mortality between the two study cohorts by using a Cox proportional hazard model, adjusted for age, sex, unprovoked or secondary deep venous thrombosis, study site, and the use of oral anticoagulants during the study period. Finally, we applied an independent samples $t$ test to compare within patient changes in quality of life scores from baseline to time of last follow-up and a Mann-Whitney U test to compare quality of life between the two patient groups at the end of follow-up.

After locking the database, we noted that $28 \%$ of patients had been incorrectly treated with $23-32 \mathrm{~mm} \mathrm{Hg}$ graduated active stockings instead of the intended ECS class of 34-46 mm Hg. To correct for this potential confounder, we calculated the effect of ECS class on the primary endpoint and additionally repeated all secondary analyses with adjustment for ECS class.

We used SPSS statistics 22.0 for all statistical analyses. $\mathrm{P}$ values below 0.05 were considered statistically significant.

\section{Patient involvement}

No patients were involved in setting the research question or the outcome measures, nor were they involved in developing plans for recruitment, design, or implementation of the study. No patients were asked to advise on interpretation or writing up of results. The results of the research will be disseminated to the relevant patient community after publication.

\section{Results}

\section{Patients}

Figure 2 shows the flow chart of the study, mapping the inclusion and progress of participants. Of 3603 
eligible patients, 1110 declined to participate and 1971 were excluded. Reasons for exclusion were previous deep venous thrombosis in the same leg as the index event $(n=530)$, calf vein thrombosis only $(n=527)$, other indication for use of compression stockings ( $n=140)$, or post-thrombotic syndrome diagnosed in the first year after deep venous thrombosis $(n=93)$. Furthermore, 56 patients had an expected lifespan of less than six months, 293 died in the first year after deep venous thrombosis before randomisation, and 332 had serious comorbid conditions or were geographically inaccessible precluding follow-up. Finally, four ineligible patients were incorrectly randomised (one patient diagnosed as having post-thrombotic syndrome at baseline and three patients who did not use ECS at the time of randomisation) and were excluded, leaving 518 patients for the primary analysis, of whom 256 were allocated to the group who stopped using ECS and 262 to the group who continued use of ECS. Baseline characteristics were comparable between the two groups with regard to age, sex, previous history of venous thromboembolism, cause of index deep venous thrombosis, mean duration of anticoagulation, number of patients on active anticoagulant treatment at randomisation, and frequency of ECS use at baseline (table 1).

\section{Study flow}

During the study period, 12 patients were lost to follow-up (six (2.3\%) in the stop-ECS group and six (2.3\%) in the continue-ECS group) and 19 patients withdrew informed consent (13 (5.1\%) v 6 (2.3\%) (fig 2). The 12 patients lost to follow-up included two patients who moved out of the area (one patient in each group). Of the 19 patients who withdrew consent, 13 failed to comply with the treatment allocation: two patients did not want to stop using ECS after being randomised to the stop-ECS group, six started using ECS during the follow-up despite being allocated to stop wearing ECS, and five from the continue-ECS group stopped using ECS during the follow-up. Thus, 518 patients (256 in the stop-ECS group and 262 in the continue-ECS group) were available for the primary efficacy analysis.

\section{Primary endpoint}

At the 12 month follow-up, data on adherence to ECS was available for 218 patients in the continue-ECS group. In this group, 186 (85\%) patients reported use of ECS six to seven days a week, $15(7 \%)$ reported use four to five days a week, and 17 (8\%) reported use less than four days a week. In the stop-ECS group, 51 patients developed post-thrombotic syndrome compared with 34 patients in the continue-ECS group. The incidence of post-thrombotic syndrome in the second year after deep venous thrombosis was $19.9 \%$ (95\% confidence interval $16 \%$ to $24 \%$ ) in the stop-ECS group and $13.0 \%$ (9.9\% to $17 \%$ ) in the continue-ECS group, for an absolute difference of $6.9 \%$ (95\% confidence interval upper limit $12.3 \%$ ) (table 2). Hence, we did not meet our predefined criterion for non-inferiority, as the upper $95 \%$ confi-

\begin{tabular}{|c|c|c|}
\hline Characteristics & Stop-ECS $(n=256)$ & Continue-ECS $(n=262)$ \\
\hline Mean (SD) age, years & $57(14)$ & $56(14)$ \\
\hline Male sex & $152(59)$ & $155(59)$ \\
\hline Mean (SD) body mass index & $28(4.8)$ & $28(4.5)$ \\
\hline Median (IQR) duration of complaints before diagnosis of DVT, days & $5(2-14)$ & $5(2-14)$ \\
\hline History of venous thromboembolism & $37(15)$ & $38(15)$ \\
\hline Provoked index DVT & $142(56)$ & $161(62)$ \\
\hline Trauma $<8$ weeks & $57(22)$ & $61(23)$ \\
\hline Surgery (general anaesthesia) $<8$ weeks & $32(13)$ & $33(13)$ \\
\hline Prolonged immobilisation $>6$ days & $26(10)$ & $22(8)$ \\
\hline Pregnant, postpartum, hormonal replacement therapy & $55(22)$ & $64(24)$ \\
\hline Cancer active at diagnosis & $29(11)$ & $21(8)$ \\
\hline Known thrombophilia* & $17(7)$ & $24(9)$ \\
\hline History of varicose veins & $36(14)$ & $29(11)$ \\
\hline Current or former smoking & $67(26)$ & $68(26)$ \\
\hline Index DVT in left leg & $158(62)$ & $144(55)$ \\
\hline \multicolumn{3}{|l|}{ Most proximal location of DVT: } \\
\hline Iliac vein & $23(9)$ & $25(10)$ \\
\hline Communal femoral vein & $44(17)$ & $31(12)$ \\
\hline Superficial femoral vein & $58(23)$ & $54(21)$ \\
\hline Popliteal vein & $131(51)$ & $152(58)$ \\
\hline \multicolumn{3}{|l|}{ Treatment of DVT: } \\
\hline Median (IQR) duration of LMWH or heparin in patients switched to VKA, days & $10(7-21)$ & $12(7-21)$ \\
\hline Median (IQR) duration of VKA treatment, months & $6(4-6)$ & $6(4.5-6)$ \\
\hline Active anticoagulant treatment at randomisation & $39(15)$ & $37(14)$ \\
\hline Frequent users of ECS at baseline ( $\geq 6$ days/week) & $232(91)$ & $249(95)$ \\
\hline Mean (SD) baseline Villalta score & $1.9(1.3)$ & $1.8(1.3)$ \\
\hline
\end{tabular}




\begin{tabular}{|c|c|c|c|}
\hline & Stop-ECS group $(n=256)$ & Continue-ECS group $(n=262)$ & Adjusted hazard ratio $(95 \% \mathrm{Cl}) / \mathrm{P}$ value \\
\hline \multicolumn{4}{|l|}{ Primary endpoint } \\
\hline PTS incidence, \% (95\% Cl) & 19.9 (16 to 24$)$ & $13.0(9.9$ to 17$)$ & $1.6(1.02 \text { to } 2.5)^{\star}$ \\
\hline Absolute No (\%) of mild PTS (Villalta scale 5-9) & $43(84)$ & $31(91)$ & - \\
\hline Absolute No (\%) of moderate PTS (Villalta scale 10-14) & $8(16)$ & $2(6)$ & - \\
\hline Absolute No (\%) of severe PTS (Villalta scale >14) & 0 & $1(3)$ & - \\
\hline \multicolumn{4}{|l|}{ Secondary endpoints } \\
\hline Incidence of overall mortality, \% (95\% Cl) & $0.78(0.003$ to 3.0$)$ & $0(0$ to 1.7$)$ & $0.97(\mathrm{NC}) \dagger$ \\
\hline Incidence of ipsilateral recurrent DVT, \% (95\% CI) & $3.1(1.5$ to 6.2$)$ & $2.3(0.94$ to 5.0$)$ & $1.5(0.54$ to 4.2$) \ddagger$ \\
\hline \multicolumn{4}{|l|}{ Quality of life: } \\
\hline Median (IQR) VEINES-QOL at end of follow-up & $96(92-98)$ & $96(90-99)$ & $P=0.99 \S$ \\
\hline Median (IQR) VEINES-SYM at end of follow-up & $95(88-100)$ & $95(88-100)$ & $P=0.99 \S$ \\
\hline Mean $(95 \% \mathrm{Cl})$ intra-individual change in VEINES-QOL & $-0.15(-1.5$ to 1.2$)$ & $0.93(-0.29$ to 2.1$)$ & $P=0.21 \uparrow$ \\
\hline Mean $(95 \% \mathrm{CI})$ intra-individual change in VEINES-Sym & $-1.1(-1.7$ to 0.44$)$ & $0.58(-0.90$ to 2.1$)$ & $P=0.12 \uparrow$ \\
\hline \multicolumn{4}{|c|}{$\begin{array}{l}\text { DVT=deep venous thrombosis; IQR=interquartile range; PTS=post-thrombotic syndrome. } \\
\text { ^Adjusted for age, sex, body mass index, unprovoked or secondary DVT, extent of index DVT, baseline Villalta score, and use of oral anticoagulants during study period. } \\
\text { †Not computable owing to } 0 \text { cases in continue-ECS group. } \\
\text { fAdjusted for age, sex, unprovoked or secondary DVT, and use of oral anticoagulants during study period. } \\
\text { §Mann-Whitney U test. } \\
\text { १Independent samples } t \text { test. }\end{array}$} \\
\hline
\end{tabular}

dence limit of the absolute difference in the incidence of post-thrombotic syndrome exceeded the $10 \%$ noninferiority limit (as shown in fig 3).

The number needed to treat to prevent one case of post-thrombotic syndrome by continuing ECS was 14 (95\% confidence interval lower limit 8). The median period between randomisation and occurrence of post-thrombotic syndrome was 4 (interquartile range 3-6) months in the stop-ECS group compared with 6 (3-12) months in the continue-ECS group ( $\mathrm{P}=0.17$ by Mann-Whitney U test). Of the 51 patients in the stop-ECS group who developed post-thrombotic syndrome, 43 (84\%) were classified as mild (Villalta score 5-9), eight $(16 \%)$ as moderate (Villalta score 10-14), and none as severe post-thrombotic syndrome (ulcer or Villalta score $>14)$ compared with $31(91 \%), 2(6 \%)$, and $1(3 \%)$ in the continue-ECS group ( $\mathrm{P}=0.19$ by $\chi^{2}$ test) (table 2$)$. The one patient with severe post-thrombotic syndrome was diagnosed as having a venous ulcer. The adjusted hazard ratio for development of post-thrombotic syndrome was 1.6 (95\% confidence interval 1.02 to 2.5 ) to the disadvantage of patients in the stop-ECS group.

\section{Secondary endpoints}

The per protocol analysis yielded similar results to the intention to treat analysis, with a incidence of post-thrombotic syndrome of $19.1 \%$ (15\% to $24 \%)$ in the stop-ECS group and $12.0 \%$ (9\% to $16 \%$ ) in the contin-

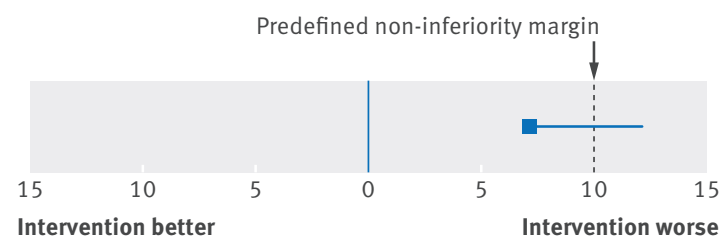

Intervention: "Stopping ECS 12 months after DVT"

Fig 3 | Treatment difference outcome with non-inferiority margin. DVT=deep venous thrombosis; $E C S=$ elastic compression stockings
ue-ECS group, for an absolute difference of 7.1\% (95\% confidence interval upper limit 13\%). Again, this did not meet our predefined criterion for non-inferiority, as the upper $95 \%$ confidence limit for the absolute difference in the incidence of post-thrombotic syndrome was 13\% in the per-protocol analysis, which is greater than the 10\% upper limit for non-inferiority. The adjusted hazard ratio for development of post-thrombotic syndrome was 1.4 (0.86 to 2.0) to the disadvantage of patients in the stop-ECS group.

Fourteen patients developed recurrent ipsilateral deep venous thrombosis: eight (3.1\%) in the stop-ECS group and six $(2.3 \%)$ in the continue-ECS group (table 2). Two patients in the stop-ECS group died (incidence $0.78 \%$ ), compared with none in the continue-ECS group. Causes of death were end stage pulmonary carcinoma (male, aged 73) and heart failure after myocardial infarction leading to asystole (female, aged 75).

At baseline, 253 (99\%) of 256 patients in the stop-ECS group and 259 (99\%) of 262 patients in the continue-ECS group completed the VEINES-QOL/Sym questionnaire, compared with 186 ( $81 \%$ of 230 available patients) and 212 ( $87 \%$ of 245 available patients) at the end of follow-up. In the stop-ECS group, VEINES-QOL and VEINES-Sym scores decreased non-significantly, whereas both improved slightly in the continue-ECS group during the follow-up period (table 2). We found no statistical differences between the two study groups with regards to the intra-individual changes in both dimensions, nor in the median quality of life at the end of follow-up.

ECS class was not associated with the occurrence of the primary endpoint (hazard ratio $0.93,0.58$ to 1.5 ). Adjustment for ECS class did not change the secondary endpoints, including quality of life.

\section{Discussion}

Post-thrombotic complaints often occur in patients with proximal deep venous thrombosis. We have shown that after an episode of proximal deep venous thrombosis, stopping ECS therapy failed to meet the criterion for 
non-inferiority compared with the current recommended medical practice of continuing to wear stockings for a second year among patients who completed one year of compliant use of elastic compression stockings. We observed an absolute excess in diagnoses of post-thrombotic syndrome of 6.9 percentage points ( $95 \%$ confidence interval upper limit 12.3\%) in the group that stopped using ECS after one year, although the mean severity of post-thrombotic syndrome did not differ between the two groups. Importantly, only $16 \%$ of patients in the stop-ECS group developed moderate or severe post-thrombotic syndrome in the second year. ECS did not affect the rate of recurrent ipsilateral deep venous thrombosis nor venous disease specific quality of life.

In the per protocol analysis, the difference between the active treatment and no ECS therapy was comparable, with the upper limit of the $95 \%$ confidence interval exceeding the non-inferiority limit of $10 \%$.

\section{Comparison with previous studies}

Our results seem to be in contrast to those of the recently published SOX trial showing that ECS did not prevent post-thrombotic syndrome after a first proximal deep venous thrombosis, although the primary endpoint in the studies was different precluding a direct comparison..$^{10}$ In that study, 806 patients were randomised to wear active (30-40 $\mathrm{mm} \mathrm{Hg}$ ) versus placebo ( $<5 \mathrm{~mm} \mathrm{Hg}$ compression at the ankle) ECS for a two year period. The primary endpoint-cumulative incidence of post-thrombotic syndrome assessed by Ginsberg's criteria-was $14.2 \%$ for active ECS compared with $12.7 \%$ in the placebo ECS group, for a non-significant hazard ratio of 1.13 (95\% confidence interval 0.73 to 1.76$).{ }^{10}$ The Villalta score was used as a secondary outcome measure in this study. The cumulative incidences of post-thrombotic syndrome measured by Villalta's criteria in that study were $52.6 \%$ and $52.3 \%$, respectively. Notably, adherence in that study was much lower than in our study, with only $56 \%$ of patients wearing their stocking for at least three days a week at the end of the follow-up period. In contrast, $93 \%$ of the patients in our study reported use of ECS at least six days a week at baseline. At the end of follow-up in our continue-ECS group, $84 \%$ of patients reported using ECS six to seven days a week, and $94 \%$ of patients used the stockings for at least four days a week. The threshold for frequent ECS use of three days a week in the SOX trial may have been too lenient to detect an effect of ECS on development of post-thrombotic syndrome. Another difference between the two studies was that our study was not placebo controlled, which may also have contributed to the difference in study results, although we consider the factor of adherence to be more important.

Our results are in line with those of three other randomised controlled trials. ${ }^{4624}$ Two single centre, open label studies randomised patients with proximal deep venous thrombosis to either daily use of ECS for at least two years or no stockings. ${ }^{46}$ Both studies reported a beneficial effect of ECS with an absolute reduction in the cumulative post-thrombotic syndrome incidence as measured with the Villalta or a Villalta-like scale of more than $20 \%$. A third small trial randomised patients with deep venous thrombosis who had used ECS for six months to stopping stockings or using them for an additional 18 months. ${ }^{24} \mathrm{~A}$ non-significant reduction in post-thrombotic syndrome of 6.9 percentage points was observed in the patients allocated to continued ECS use. Notably, post-thrombotic syndrome in this study was assessed according to the CEAP classification, which combines clinical and objective findings but does not take symptoms into account, in contrast to Villalta's scale. Symptom relief, which was a secondary outcome of the study, was significantly in favour of active use of ECS during the first year but not thereafter. ${ }^{24}$ Self reported adherence to the therapy in all three studies was excellent, with more than $90 \%$ of patients wearing the stockings for at least six days a week. A limitation of all three studies is the lack of a double blind design. With this in mind, the number needed to treat increases from five in the first year to eight in the second year with a range from seven to 62 .

\section{Strengths and limitations of study}

Strengths of our study include its multicentre design, the high quality centralised randomisation procedure, the considerable sample size, the very good adherence to the allocated therapy and response rates for the quality of life questionnaires, the exclusion of patients with deep venous thrombosis limited to the distal veins, and the assessment of both post-thrombotic syndrome and quality of life at several time points during follow-up.

Some methodological considerations of our study require comment. Only patients who were committed to dedicated use of the stockings were included in our study. Also, patients who had already developed post-thrombotic syndrome in the first year after deep venous thrombosis were ineligible to participate. Therefore, the study conclusions cannot necessarily be generalised to all patients with proximal deep venous thrombosis. Even so, this specific design allowed us to study the "true" effect of ECS without confounding by increasingly diminishing adherence throughout the follow-up period. With our design, we achieved excellent adherence with only $7.3 \%$ of patients in the continue-ECS group using their stockings less than four days a week at the end of the study. Also, we did a single blinded randomised trial that was not controlled by a placebo intervention. A double blind placebo controlled study design would not have been viable because the nature of the intervention precluded effective blinding of patients through placebo stockings, as they all had 12 months' experience with active ECS. Although we used several strategies to reduce unmasking of treatment assignment to the observer who assessed the presence and severity of post-thrombotic syndrome, such as repeated strict instructions to the study participants not to reveal their allocation and not to wear the stocking on the day of follow-up, we cannot exclude bias caused by possible loss of blinding. Also, as use of stockings and some baseline variables such as "duration of symptoms" were reported by patients, recall bias may have been present. Furthermore, 28\% of 
patients were treated with lower grade ECS than prescribed by the protocol. Even so, post hoc analyses did not show any interaction with ECS grade for either the primary or secondary endpoints, including quality of life. Moreover, it may be assumed that if the less effective than intended ECS treatment did alter our primary endpoint, our results represent an underestimation of the "true" therapeutic effect, which would underline our conclusion.

Another limitation of our study is that $19(3.6 \%)$ patients withdrew their consent and $12(2.3 \%)$ patients were lost to follow-up. This is below the estimated $10 \%$ in the power calculation and half the number reported in a recently published study in this field of research. ${ }^{10}$ Finally, we acknowledge that the rate of post-thrombotic syndrome in our study was higher than anticipated in the sample size considerations, which may have resulted in a lower power to prove our non-inferiority hypothesis.

\section{Conclusions}

We conclude that, in patients with a proximal deep venous thrombosis who are motivated to wear compression stockings, a strategy to stop ECS therapy after one year is not non-inferior to completing a two year period of ECS therapy, as the former is associated with an excess in diagnoses of post-thrombotic syndrome in the second year. Our results suggest that ECS affect the natural history of development of post-thrombotic syndrome after deep venous thrombosis. However, quality of life did not differ and only a few patients developed moderate or severe post-thrombotic syndrome in the second year in both study groups. On the basis of our results, we recommend continuing ECS therapy for 24 months after deep venous thrombosis. Of note, it may be hypothesised that prolonging ECS use after the 24 month period may be beneficial to selected patients to continuously prevent post-thrombotic syndrome development, as post-thrombotic changes are irreversible. Obviously, this will need to be tested in future trials.

\section{AUTHOR AFFILIATIONS}

Department of Internal Medicine, Diakonessenhuis Hospital, Utrecht, Netherlands

2Department of Internal Medicine, University Medical Center, Utrecht, Netherlands

${ }^{3}$ Department of Vascular Medicine, University Medical Center, 3508 GA Utrecht, Netherlands

${ }^{4}$ Department of Thrombosis and Hemostasis, Leiden University Medical Center, Leiden, Netherlands

${ }^{5}$ Department of Dermatology, Diakonessenhuis Hospital, Utrecht, Netherlands

${ }^{6}$ Department of Radiology, Diakonessenhuis Hospital, Utrecht, Netherlands

'Department of Dermatology, St Antonius Hospital, Nieuwegein, Netherlands

${ }^{8}$ Department of Hematology, St Antonius Hospital, Nieuwegein, Netherlands

${ }^{9}$ Department of Internal Medicine, Groene Hart Hospital, Gouda, Netherlands

${ }^{10}$ Department of Internal Medicine, Rijnstate Hospital, Arnhem, Netherlands

${ }^{11}$ Department of Hematology, Reinier de Graaf Group, Delft, Netherlands
${ }^{12}$ Department of Internal Medicine, Gelre Hospitals, Apeldoorn, Netherlands

${ }^{13}$ Department of Dermatology, Gelderse Vallei Hospital, Ede, Netherlands

We thank all the patients and study personnel who helped in the conduct of this study.

Contributors: GCM, MAvdR, and MVH designed the study, analysed the data, and wrote the manuscript. FAK analysed the data and wrote the manuscript. MJAMT, FBMS, MMCH, and DHB were involved in study design. GCM, MAvdR, SK, OdW, TK, MMCH, HAHK, REB, EK, CGS, WS, and WPA included patients. All authors critically reviewed and approved the final manuscript. GCM is the guarantor.

Funding: Fonds NutsOhra, Stichting Voorzorg, the Cornelis Visser Stichting, Sanofi (unrestricted grant), and the Stichting Medicina et Scientia in the Netherlands provided research grants. All analyses were performed independently from the funding source.

Competing interests: All authors have completed the ICMJE uniform disclosure form at www.icmje.org/coi_disclosure.pdf and declare: no support from any organisation for the submitted work other than that described above; no financial relationships with any organisations that might have an interest in the submitted work in the previous three years; no other relationships or activities that could appear to have influenced the submitted work.

Ethical approval: Approval from the central institutional research and ethics board (VCMO, Nieuwegein, the Netherlands) was obtained by all participating centres. All patients provided written informed consent.

Data sharing: No additional data available.

Transparency: The lead author affirms that this manuscript is an honest, accurate, and transparent account of the study being reported; that no important aspects of the study have been omitted; and that any discrepancies from the study as planned (and, if relevant, registered) have been explained.

This is an Open Access article distributed in accordance with the Creative Commons Attribution Non Commercial (CC BY-NC 3.0) license, which permits others to distribute, remix, adapt, build upon this work non-commercially, and license their derivative works on different terms, provided the original work is properly cited and the use is non-commercial. See: http://creativecommons.org/licenses/ by-nc/3.0/.

1 Kahn SR, Shrier I, Julian JA, et al. Determinants and time course of the postthrombotic syndrome after acute deep venous thrombosis. Ann Intern Med 2008;149:698-707. doi:10.7326/0003-4819-149-10-200811180-00004

2 Kahn SR, Comerota AJ, Cushman M, et al. American Heart Association Council on Peripheral Vascular Disease, Council on Clinical Cardiology, and Council on Cardiovascular and Stroke Nursing. The postthrombotic syndrome: evidence-based prevention, diagnosis, and treatment strategies: a scientific statement from the American Heart Association. Circulation 2014;130:1636-61. doi:10.1161/ CIR.0000000000000130

3 Saarinen J, Kallio T, Lehto M, Hiltunen S, Sisto T. The occurrence of the post-thrombotic changes after an acute deep venous thrombosis. A prospective two-year follow-up study. J Cardiovasc Surg (Torino) 2000;41:441-6.

4 Brandjes DP, Büller HR, Heijboer H, et al. Randomised trial of effect of compression stockings in patients with symptomatic proximal-vein thrombosis. Lancet 1997;349:759-62. doi:10.1016/ S0140-6736(96)12215-7.

5 Kahn SR, Ginsberg JS. Relationship between deep venous thrombosis and the postthrombotic syndrome. Arch Intern Med 2004;164:17-26. doi:10.1001/archinte.164.1.17.

6 Prandoni P, Lensing AW, Prins MH, et al. Below-knee elastic compression stockings to prevent the post-thrombotic syndrome: a randomized, controlled trial. Ann Intern Med 2004;141:249-56. doi:10.7326/0003-4819-141-4-200408170-00004

7 Baldwin MJ, Moore HM, Rudarakanchana N, Gohel M, Davies AH. Post-thrombotic syndrome: a clinical review. J Thromb Haemost 2013;11:795-805. doi:10.1111/jth.12180.

8 Kahn SR, Shbaklo H, Lamping DL, et al. Determinants of health-related quality of life during the 2 years following deep vein thrombosis. J Thromb Haemost 2008;6:1105-12. doi:10.1111/j.1538-7836.2008.03002.x.

9 Guanella R, Ducruet T, Johri M, et al. Economic burden and cost determinants of deep vein thrombosis during 2 years following diagnosis: a prospective evaluation. J Thromb Haemost 2011;9:2397405. doi:10.1111/j.1538-7836.2011.04516.x.

10 Kahn SR, Shapiro S, Wells PS, et al. SOX trial investigators. Compression stockings to prevent post-thrombotic syndrome: a randomised placebo-controlled trial. Lancet 2014;383:880-8. doi:10.1016/S0140-6736(13)61902-9. 
11 Prandoni P, Villalta S, Bagatella P, et al. The clinical course of deep-vein thrombosis. Prospective long-term follow-up of 528 symptomatic patients. Haematologica 1997;82:423-8.

12 ten Cate-Hoek AJ, ten Cate H, Tordoir J, Hamulyák K, Prins MH. Individually tailored duration of elastic compression therapy in relation to incidence of the postthrombotic syndrome. I Vasc Surg 2010;52:132-8. doi:10.1016/i.jvs.2010.01.089.

13 ten Cate-Hoek AJ, Bouman AC, Joore MA, Prins M, Ten Cate H. IDEAL DVT trial investigators. The IDEAL DVT study, individualised duration elastic compression therapy against long-term duration of therapy for the prevention of post-thrombotic syndrome: protocol of a randomised controlled trial. BMJ Open 2014:4:e005265. doi:10.1136/ bmjopen-2014-005265.

14 Villalta S, Bagatella P, Piccioli A, et al. Assessment of validity and reproducibility of a clinical scale for the post-thrombotic syndrome. Haemostasis 1994;24:158a.

15 Kahn SR, Partsch $\mathrm{H}$, Vedantham S, Prandoni P, Kearon C. Subcommittee on Control of Anticoagulation of the Scientific and Standardization Committee of the International Society on Thrombosis and Haemostasis. Definition of post-thrombotic syndrome of the leg for use in clinical investigations: a recommendation for standardization. / Thromb Haemost 2009;7:87983. doi:10.1111/j.1538-7836.2009.03294.x.

16 Kahn SR. Measurement properties of the Villalta scale to define and classify the severity of the post-thrombotic syndrome. I Thromb Haemost 2009;7:884-8. doi:10.1111/j.1538-7836.2009.03339.x.

17 Kahn SR, Lamping DL, Ducruet T, et al. VETO Study investigators. VEINES-QOL/Sym questionnaire was a reliable and valid diseasespecific quality of life measure for deep venous thrombosis. J Clin Epidemiol 2006:59:1049-56. doi:10.1016/i.jclinepi.2005.10.016.
18 Lamping DL, Schroter S, Kurz X, Kahn SR, Abenhaim L. Evaluation of outcomes in chronic venous disorders of the leg: development of a scientifically rigorous, patient-reported measure of symptoms and quality of life. J Vasc Surg 2003;37:410-9. doi:10.1067/ mva.2003.152

19 Catarinella F, Nieman F, de Wolf M, Wittens C. Short-term follow-up of Quality-of-Life in interventionally treated patients with postthrombotic syndrome after deep venous occlusion. Phlebology 2014:29(suppl):104-11. doi:10.1177/0268355514529505.

20 Catarinella FS, Nieman FH, de Wolf MA, Toonder IM, de Graaf R, Wittens CH. Quality-of-life in interventionally treated patients with post-thrombotic syndrome. Phlebology 2015;30(Suppl):89-94. doi:10.1177/0268355515569431.

21 Kearon C, Ginsberg JS, Hirsh J. The role of venous ultrasonography in the diagnosis of suspected deep venous thrombosis and pulmonary embolism. Ann Intern Med 1998;129:1044-9. doi:10.7326/0003-4819-129-12-199812150-00009.

22 Prandoni P, Cogo A, Bernardi E, et al. A simple ultrasound approach for detection of recurrent proximal-vein thrombosis. Circulation 1993;88:1730-5. doi:10.1161/01.CIR.88.4.1730.

23 Tan M, Mol G, Kees van Rooden J, Huisman MV. The diagnostic management of recurrent deep vein thrombosis and pulmonary embolism. Semin Respir Crit Care Med 2012;33:151-5. doi:10.1055/s-0032-1311798.

24 Aschwanden M, Jeanneret C, Koller MT, Thalhammer C, Bucher HC, Jaeger KA. Effect of prolonged treatment with compression stockings to prevent post-thrombotic sequelae: a randomized controlled trial. J Vasc Surg 2008;47:1015-21. doi:10.1016/j.jvs.2008.01.008

(c) BMJ Publishing Group Ltd 2016 